\title{
Adherence to Recommended Inpatient Hepatic Encephalopathy Workup
}

\author{
Dennis Kumral, MD*; Rehan Qayyum, MD, MHS²; Susan Roseff, MD; ${ }^{3}$ Richard K Sterling, MD, MSc'; Mohammad S Siddiqui, MD 1 \\ 'Division of Gastroenterology and Hepatology, Virginia Commonwealth University, Richmond, Virginia; ${ }^{2}$ Division of Hospital Medicine, Virginia \\ Commonwealth University, Richmond, Virginia; ${ }^{3}$ Department of Pathology, Virginia Commonwealth University, Richmond, Virginia.
}

Hepatic encephalopathy (HE) is characterized by altered sensorium and is the most common indication for hospitalization among patients with cirrhosis. Liver societal guidelines for inpatient HE revolve around identification of potential precipitants. In this retrospective study, we aimed to determine adherence to societal guidelines for evaluation of HE in 78 inpatients. The adherence rate to societal recommended guidelines for workup of HE was low, with only $17(22 \%)$ patients having complete diagnostic workup within 24 hours of admission. Notably, 23 (30\%) patients were not subjected to blood culture analysis, $16(21 \%)$ were missing urinalysis, and 15 (20\%) were missing chest radiograph. In patients with ascites $(\mathrm{N}=34), 26(77 \%)$ did not have a diagnostic paracentesis to exclude spontaneous bacterial peritonitis. In contrast, serum ammonia determination, a laboratory test not endorsed by societal guidelines for workup of HE, was ordered in 74 (95\%) patients. These findings underscore the limited adherence to societal guidelines in hospitalized patients with HE. Journal of Hospital Medicine 2019;14:157-160. (@ 2019 Society of Hospital Medicine

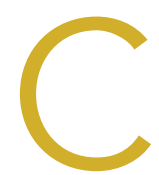

linical guidelines are periodically released by medical societies with the overarching goal of improving deliverable medical care by standardizing disease management according to best available published literature and by reducing healthcare expenditure associated with unnecessary and superfluous testing. ${ }^{1}$ Unfortunately, nonadherence to guidelines is common in clinical practice ${ }^{2}$ and contributes to the rising cost of healthcare. ${ }^{3}$ Health resource utilization is particularly relevant in management of cirrhosis, a condition with an annual healthcare expenditure of $\$ 13$ billion. ${ }^{4}$ Hepatic encephalopathy $(\mathrm{HE})$, the most common complication of cirrhosis, is characterized by altered sensorium and is the leading indication for hospitalization among cirrhotics. The joint guidelines of the European Association for the Study of the Liver (EASL) and the American Association for the Study of Liver Diseases (AASLD) for diagnostic workup for HE recommend identification and treatment of potential precipitants. ${ }^{5}$ The guidelines also recommend against checking serum ammonia levels, which have not been shown to correlate with diagnosis or severity of HE. ${ }^{6-8}$ Currently, limited data are available on practice patterns regarding guideline adherence and unnecessary serum ammonia testing for initial evaluation of $\mathrm{HE}$ in hospitals. To overcome this gap in knowledge, we conducted the present study to provide granular details regarding the diagnostic workup for hospitalized patients with HE.

*Corresponding Author: Dennis Kumral, M.D.; E-mail: dennis.kumral1@ vcuhealth.org; Telephone: 808-828-4060.

Received: August 21, 2018; Revised: December 6, 2018;

Accepted: December 20, 2018

๑ 2019 Society of Hospital Medicine DOI 10.12788/jhm.3152

\section{METHODS}

This study adopted a retrospective design and recruited patients admitted to the Virginia Commonwealth University Medical Center between July 1, 2016 and July 1, 2017. The institutional review board approved the study, and the manuscript was reviewed and approved by all authors prior to submission. All chart reviews were performed by hepatologists with access to patients' electronic medical record (EMR).

\section{Patient Population}

Patients were identified from the EMR system by using ICD-9 and ICD-10 codes for cirrhosis, hepatic encephalopathy, and altered mental status. All consecutive admissions with these diagnosis codes were considered for inclusion. Adult patients with cirrhosis resulting from any etiology of chronic liver diseases with primary reason for admission of HE were included. If patients were readmitted for HE during the study period, then only the data from index HE admission was included in the analysis and data from subsequent admissions were excluded. The other exclusion criteria included non-HE causes of confusion, acute liver failure, and those admitted with a preformulated plan (eg, direct hepatology clinic admission or outside hospital transfer). Patients who developed HE during their hospitalization where HE was not the indication for admission were also excluded. Finally, all patients admitted under the direct care of hepatology were excluded.

\section{Diagnostic Workup}

The recommendations of the AASLD and the EASL for workup for HE include obtaining detailed history and physical examination supplemented by diagnostic evaluation for potential HE precipitants including infections, electrolyte disturbances, dehydration, renal failure, glycemic disturbances, and toxin 
TABLE. Baseline Clinical Characteristics of Patients ( $\mathrm{N}=78)$ Admitted with Hepatic Encephalopathy

\begin{tabular}{|c|c|c|c|c|}
\hline Characteristic & $\begin{array}{l}\text { Entire Cohort } \\
\qquad(\mathrm{N}=78)\end{array}$ & $\begin{array}{l}\text { Complete Workup } \\
\qquad(\mathrm{n}=17)\end{array}$ & $\begin{array}{l}\text { Incomplete Workup } \\
\qquad(\mathrm{n}=61)\end{array}$ & $P$ Value \\
\hline Age (years) & $59.3 \pm 9$ & $60.1 \pm 11$ & $59.1 \pm 8$ & .36 \\
\hline Male gender (\%) & $53(68)$ & $13(77)$ & $40(66)$ & .56 \\
\hline Ethnicity (\%) & & & & .53 \\
\hline Caucasian & $54(69)$ & $10(59)$ & $44(72)$ & \\
\hline Black & $22(28)$ & $7(41)$ & $15(25)$ & \\
\hline Other & $2(23)$ & $0(0)$ & $2(3)$ & \\
\hline BMI (kg/m2) & $28.8 \pm 7$ & $29.8 \pm 10$ & $28.5 \pm 6$ & .49 \\
\hline Etiology of Cirrhosis (\%) & & & & .52 \\
\hline Hepatitis C & $41(53)$ & $9(53)$ & $32(53)$ & \\
\hline Alcohol & $14(18)$ & $4(24)$ & $10(16.4)$ & \\
\hline Nonalcoholic steatohepatitis & $13(17)$ & $2(12)$ & $11(18)$ & \\
\hline Other & $10(13)$ & $2(12)$ & $8(19)$ & \\
\hline \multicolumn{5}{|l|}{ Complications of Cirrhosis (\%) } \\
\hline History of HE & $53(68)$ & $13(77)$ & $40(66)$ & .56 \\
\hline Ascites & $34(44)$ & $0(0)$ & $34(56)$ & $<.001$ \\
\hline Esophageal Varices & $42(54)$ & $10(59)$ & $32(53)$ & .79 \\
\hline TIPS & $14(18)$ & $3(18)$ & $11(18)$ & 1.00 \\
\hline Hepatocellular carcinoma & $4(5)$ & $2(12)$ & $2(3)$ & .21 \\
\hline \multicolumn{5}{|l|}{ Pertinent Laboratory Values } \\
\hline AST (IU/L) & $79.1 \pm 52$ & $111.7 \pm 75$ & $71 \pm 39$ & $<.01$ \\
\hline ALT (IU/L) & $41.2 \pm 33$ & $57.4 \pm 55$ & $36.8 \pm 22$ & .02 \\
\hline Bilirubin (mg/dL) & $3.3 \pm 5$ & $5.4 \pm 10$ & $2.8 \pm 2$ & .05 \\
\hline Albumin (g/mL) & $2.9 \pm 1$ & $2.9 \pm 1$ & $2.9 \pm 1$ & .64 \\
\hline Creatinine (mg/dL) & $1.5 \pm 1$ & $1.3 \pm 1$ & $1.6 \pm 2$ & .52 \\
\hline Sodium (mg/dL) & $136.8 \pm 6$ & $136.8 \pm 4$ & $136.8 \pm 7$ & .99 \\
\hline Platelet Count (x1012) & $110.3 \pm 79$ & $101.1 \pm 80$ & $112.9 \pm 80$ & .59 \\
\hline INR & $1.7 \pm 1$ & $1.6 \pm 1$ & $1.7 \pm 0.6$ & .69 \\
\hline MELD Score & $17 \pm 8$ & $16.1 \pm 10$ & $17.0 \pm 8$ & .71 \\
\hline
\end{tabular}

Abbreviations: ALT, alanine aminotransferase; AST, aspartate aminotransferase; BMI, body mass index; HE, hepatic encephalopathy; INR, international normalized ratio; MELD, model for end stage liver disease; TIPS, intrahepatic portosystemic shunt

ingestion (eg, alcohol, illicit drugs). ${ }^{5}$ Based on the guideline recommendation, this study defined a "complete workup" as including all of the following elements: infection evaluation (blood culture, urinalysis/urine culture, chest radiograph, diagnostic paracentesis in the presence of ascites), electrolyte/ renal evaluation (serum sodium, potassium, creatinine, and glucose), and toxin evaluation (urine drug screening). Any HE admission that was missing elements from the aforementioned battery of tests was defined as "incomplete workup." In patients admitted with decompensated cirrhosis, serum ammonia testing was considered inappropriate unless there was a nuanced explanation supporting its use documented within the EMR. The frequency and specialty of the physician ordering serum ammonia level tests were determined. The financial burden of unnecessary ammonia testing was estimated by assigning a laboratory charge (\$258) for each patient.

\section{Statistical Analysis}

Continuous and categorical variables are reported as means ( \pm standard deviation), median (interquartile range or IQR), or proportion (\%) as appropriate. Across-group differences were compared using Student t-test for normally distributed continuous variables and Mann-Whitney $U$ test for skewed data. Fisher's exact test was used to compare proportion. HE evalua- 


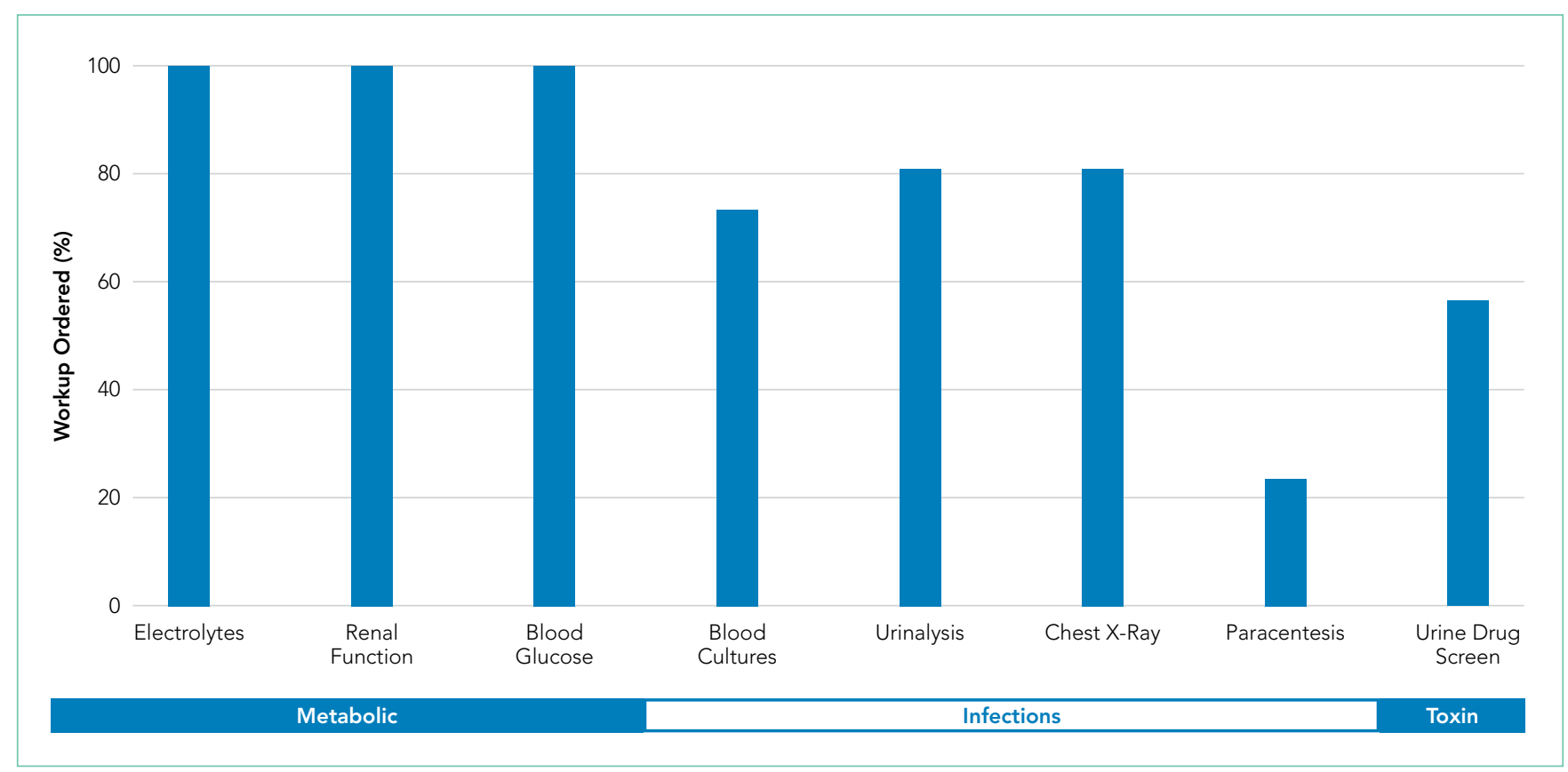

FIG. Adherence to the Recommended Diagnostic Evaluation of HE Precipitants Abbreviation: $\mathrm{HE}$, hepatic encephalopathy

tions were quantified by the number of patients with complete workup and by the number of patients with missing components of the workup. A nominal $P$ value of less than .05 was considered statistically significant. All statistical analyses were performed using SPSS Statistics version 24.0 (IBM Corporation, Armonk, New York).

\section{RESULTS}

\section{Cohort Characteristics}

The baseline cohort demographics are listed in the Table. Of the 145 patients identified using diagnostic codes for cirrhosis, 78 subjects met the study criteria. The most common exclusion criteria included non-HE etiology of altered mental status $(n=37)$ and patients with readmissions for HE during the study period $(n=30)$. The mean age of the study cohort was 59.3 years, and the most common etiology of cirrhosis was hepatitis $C(n=41)$, alcohol induced $(n=14)$, and nonalcoholic steatohepatitis $(n=13)$.

\section{Initial Diagnostic Evaluation}

The major precipitants of HE in the study cohort were ineffective lactulose dosing ( $n=43)$, infections $(n=25)$, and electrolyte disturbances/renal injury $(n=6)$. At the time of admission, 53 patients were on therapy for HE. Only 17 (22\%) patients had complete diagnostic workup within 24 hours of hospital admission. The individual components of the complete workup are shown in the Figure. Notably, 23 (30\%) patients were missing blood cultures, $16(21 \%)$ were missing urinalysis, 15 (20\%) were missing chest radiograph, and 34 (44\%) were missing urine drug screening. Of the 34 patients with ascites on admission, only eight (23\%) had diagnostic paracentesis performed on admission to rule out spontaneous bacterial peritonitis.

\section{Serum Ammonia Testing}

Serum ammonia testing was performed on 74 patients (94.9\%), and no patient met the criteria for appropriate testing. Forty patients already had a known diagnosis of HE prior to index admission. Furthermore, 10 (14\%) patients had serum ammonia testing repeated after admission without documentation in the EMR to justify repeat testing. Emergency Department (ED) physicians ordered ammonia testing in 57 cases (77\%), internists ordered the testing in 11 cases (15\%), and intensivists ordered the testing in two cases (3\%). The patient's charges for serum ammonia testing at the time of admission and for repeat testing were $\$ 19,092$ and $\$ 2,580$, respectively.

\section{DISCUSSION}

This study utilized HE in patients with decompensated cirrhosis as a framework to analyze adherence to societal guidelines. The adherence rate to AALSD/EASL recommended inpatient evaluation of $\mathrm{HE}$ is surprisingly low, and most patients are missing key essential elements of the diagnostic work up. While the diagnostic tests that are ordered as part of a panel are completed universally (renal function, electrolytes, and glucose testing), individual testing is less inclined to be ordered (blood cultures, urine culture/urinalysis, CXR, UDS) and procedural testing, such as diagnostic paracentesis, is often missed. This last finding is in line with published literature showing that $40 \%$ of patients admitted with ascites or HE did not have diagnostic paracentesis during hospital admission despite $24 \%$ reduction of inhospital mortality among patients undergoing the procedure. ${ }^{9}$

Although serum ammonia testing is not endorsed by the AASLD/EASL guidelines for $\mathrm{HE},{ }^{5}$ it is ordered nearly universally. The cost of an individual test is relatively low, but the cumulative cost of serum ammonia testing can be substantial because 
$\mathrm{HE}$ is the most common indication for hospitalization among patients with cirrhosis. ${ }^{4}$ Initiatives, such as the Choosing Wisely ${ }^{\circledR}$ campaign, encourage high-value and evidence-based care by limiting excessive and unnecessary diagnostic testing. ${ }^{10}$ The Canadian Choosing Wisely campaign specifically includes avoidance of serum ammonia testing for diagnosis of HE to provide high-value care in hepatology. ${ }^{11}$

Although the exact reasons for nonadherence to recommended HE evaluations are unclear, a potential method to mitigate excessive testing is to utilize the EMR and ordering system. ${ }^{3}$ EMR-based strategies can curb unnecessary testing in inpatient settings. ${ }^{12}$ The use of HE order sets, the inclusion of clinical decision support systems, and the restriction of access to specialized testing can be readily incorporated into the EMR to encourage adherence to guideline-based care while limiting unnecessary testing.

This study should be interpreted in the context of study limitations. Given the retrospective design of the study, salient factors in decisions behind diagnostic testing cannot be assessed. Future studies should utilize mixed-model methodology to elucidate reasons behind these decisions. The present study used a strict definition of complete workup including all the mentioned elements of the diagnostic workup for HE; however, in clinical practice, providers could be justified in not ordering certain tests if the specific clinical scenario does not lead to its use (eg, chest X-ray deferred in a patient with clear lung exam, no symptoms, or hypoxia). Similarly, UDS was included as a required element for a complete workup. While it may be ordered in a case-by-case basis to screen for illicit drug abuse, UDS is also a critical element of the workup to screen for opioid use as a precipitant of HE. Finally, considering the strict study entry criteria, we excluded repeated admissions for $\mathrm{HE}$ during the study period and therefore likely underestimate the cost burden of serum ammonia testing.

In conclusion, valuable guideline-based diagnostic testing is often missing in patients admitted for HE while serum ammonia testing is nearly universally ordered. These findings un- derscore the importance of implementing educational strategies, such as the Choosing Wisely ${ }^{\circledR}$ campaign, and EMR-based clinical decision support systems to improve health resource utilization in patients with cirrhosis and HE.

Disclosures: The authors have nothing to disclose.

\section{References}

1. Andrews EJ, Redmond HP. A review of clinical guidelines. $\mathrm{Br} J$ Surg. 2004;91:956-964. doi: 10.1002/bjs.4630

2. Arts DL, Voncken AG, Medlock S, Abu-Hanna A, van Weert HC. Reasons for intentional guideline non-adherence: a systematic review. Int J Med Inform. 2016;89:55-62. doi: 10.1016/j.jijmedinf.2016.02.009.

3. Eaton KP, Levy K, Soong C, et al. Evidence-based guidelines to eliminate repetitive laboratory testing. JAMA Intern Med. 2017;177(12):1833-1839. doi: 10.1001/jamainternmed.2017.5152.

4. Everhart J. The burden of digestive diseases in the United States. Washington D.C.: US Department of Health and Human Services, Public Health Service, National Institutes of Health. U.S. Government Printing Office; 2008:111-114

5. Vilstrup H, Amodio P, Bajaj J, et al. Hepatic encephalopathy in chronic liver disease: 2014 Practice guideline by the American Association for the Study of Liver Diseases and the European Association for the Study of Liver Diseases. Hepatology. 2014;60:715-735. doi: 10.1002/hep.27210

6. Stahl J. Studies of the blood ammonia in liver disease: Its diagnostic, prognostic, and therapeutic significance. Ann Intern Med. 1963;58:1-24.

7. Ong JP, Aggarwal A, Kreiger D, et al. Correlation between ammonia levels and the severity of hepatic encephalopathy. Am J Med. 2003;114:188-193. doi: 10.1016/S0002-9343(02)01477-8

8. Nicalao F, Efrati C, Masini A, Merli M, Attili AF, Riggio O. Role of determination of partial pressure of ammonia in cirrhotic patients with and without hepatic encephalopathy. J Hepatol. 2003;38:441-446. doi: 10.1016/S01688278(02)00436-1

9. Orman ES, Hayashi PH, Bataller R, Barritt AS 4th. Paracentesis is associated with reduced mortality in patients hospitalized with cirrhosis and ascites. Clin Gastroenterol Hepatol. 2014;12:496-503. doi: 10.1016/j.cgh.2013.08.025.

10. Cassek CK, Guest JA. Choosing wisely: helping physicians and patients make smart decisions about their care. JAMA. 2012;307:1801-1802. doi: 10.1001/jama.2012.476.

11. Choosing Wisely Canada. 2018. Five things patients and physicians should question. Available at: https://choosingwiselycanada.org/hepatology/. Accessed November 18, 2018

12. Iturrate E, Jubelt L, Volpicelli F, Hochman K. Optimize your electronic medical record to increase value: reducing laboratory overutilization. Am J Med. 2016;129:215-220. doi: 10.1016/j.amjmed.2015.09.009. 